\title{
Experimental Study of the Characteristics of Band-Passed Ultra-High Frequency Filters with Increased Protective Band in the Interest of Ensuring Electromagnetic Compatibility
}

\author{
Valery P. Berdysheva, Andrey V. Zhuravleva, \\ Mikhail A. Egorova, Nikita V. Kopylov', \\ Vladimir A. Kopylov ${ }^{c}$ and Vitaly V. Loy*c \\ ${ }^{a}$ Military Academy of Aero-Space Defence \\ named after the Marshal of Soviet Union G.K. Zhukov \\ Tver, Russian Federation \\ ${ }^{b}$ Yaroslavl Higher Military School of Air Defense \\ Yaroslavl, Russian Federation \\ 'Siberian Federal University \\ Krasnoyarsk, Russian Federation
}

\begin{abstract}
The article presents the structure of the method for the synthesis of microwave bandpass filters (BPF) on non-uniform lines, which makes it possible to select the structure and parameters of the BPF that provide the maximum length of the stop band to meet the requirements of electromagnetic compatibility (EMC) with given restrictions on the attenuation characteristics in the passband and barrier bands. and the established design and technological limitations on the drop in wave resistance and manufacturing tolerances. The results of experimental studies of the characteristics of ultrahighfrequency bandpass filters with an increased protective band in order to ensure EMC are presented.
\end{abstract}

Keywords: electromagnetic compatibility, radio electronic means, bandpass filter, stopband, passband, three-stage resonators, wave impedance drop, frequency spacing of parasitic passbands, filter parameters tolerance, field experiment.

(C) Siberian Federal University. All rights reserved

This work is licensed under a Creative Commons Attribution-Non Commercial 4.0 International License (CC BY-NC 4.0).

* Corresponding author E-mail address: 1v2009.1974@mail.ru 


\title{
Экспериментальное исследование характеристик
}

\author{
полосно-пропускающих фильтров сверхвысоких частот \\ с увеличенной защитной полосой
}

В интересах обеспечения

электромагнитной совместимости

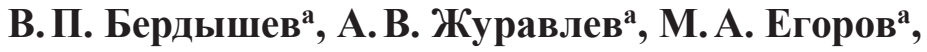

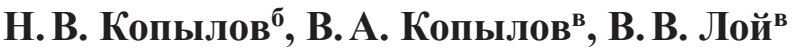 \\ ${ }^{a}$ Военная академия воздушно-космической оборонь \\ им. Маршала Советского Союза Г.К. Жукова \\ Российская Федерация, Тверь \\ бЯрославское высшее военное училище \\ противовоздушной оборонь \\ Российская Федерачия, Ярославль \\ ${ }^{8}$ Сибирский федеральныий университет \\ Российская Федерачия, Красноярск
}

\begin{abstract}
Аннотация. В статье представлена структура метода синтеза полосно-пропускающих фильтров (ППФ) СВЧ на неоднородных линиях, позволяющего осуществить выбор структуры и параметров ППФ, обеспечивающих максимальную протяженность полосы заграждения для выполнения требований электромагнитной совместимости (ЭМС) при заданных ограничениях на характеристики затухания в полосах пропускания и заграждения и установленных конструктивно-технологических ограничений по перепаду волновых сопротивлений и допускам на изготовление. Приведены результаты экспериментальных исследований характеристик полосно-пропускающих фильтров сверхвысоких частот с увеличенной защитной полосой в интересах обеспечения ЭМС.
\end{abstract}

Ключевые слова: электромагнитная совместимость, радиоэлектронные средства, полоснопропускающий фильтр, полоса заграждения, полоса пропускания, трехступенчатые резонаторы, перепад волновых сопротивлений, разнос частот паразитных полос пропускания, допуск на параметры фильтров, натурный эксперимент.

Цитирование: Бердышев, В.П. Экспериментальное исследование характеристик полосно-пропускающих фильтров сверхвысоких частот с увеличенной защитной полосой в интересах обеспечения электромагнитной совместимости / В.П. Бердышев, А. В. Журавлев, М. А. Егоров, Н. В. Копылов, В. А. Копылов, В. В. Лой // Журн. Сиб. федер. ун-та. Техника и технологии, 2021, 14(3). С. 272-280. DOI: 10.17516/1999-494X-0308

Обеспечение электромагнитной совместимости (ЭМС) радиоэлектронных средств (РЭС) включает в себя широкий комплекс мероприятий, которые подразделяют на организационные и технические. Проведение комплекса организационных мероприятий, основанных на использовании «запретов» по частоте, времени, направлению и т. д., не позволяют, особенно в военное время, качественно решать задачи ЭМС, а в мирное - приводят к снижению боевых возможностей РЭС образцов вооружения и военной техники.

Основу технических мероприятий обеспечения ЭМС составляют фильтрующие устройства (ФУ), которые определяют параметры РЭС с точки зрения помехозащищенности и ЭМС. Для борьбы с побочным излучением (ПИ) и защиты РЭС от непреднамеренных помех, воз- 
действующих по паразитным каналам приема (ПКП), наиболее эффективна частотная фильтрация, которая осуществляется в трактах высокой частоты до нелинейных преобразований сигнала с помощью полосно-пропускающих фильтров (ППФ) СВЧ.

Характеристика частотной избирательности ППФ должна обладать минимальными потерями в полосе пропускания (ПП) и заданным уровнем затухания в полосе заграждения (ПЗ). Ширина полосы заграждения оценивается разностью между ее граничными частотами. В соответствии с существующими ГОСТами и стандартами по ЭМС в зависимости от типов РЭС протяженность полосы заграждения должна составлять от 1:5 до 1:10 (разрядка ППП). Уровни затухания в ПЗ (уровни заграждения) определяются исходя, с одной стороны, из норм на ПИ и ПКП, с другой - из характеристик радиопередающих (РПДУ) и радиоприемных устройств (РПМУ) и находятся в пределах $60 \ldots 80$ дБ, а в некоторых случаях и более [1].

Анализ ФУ СВЧ, и ППФ в частности, применяемых в существующих и перспективных PTC РТВ, показывает, что они, как правило, построены на отрезках однородных линий передачи (ОЛП), которые обладают эквидистантным спектром резонансных частот. Как следствие, для этих фильтров характерно наличие паразитных полос пропускания (ППП), расположенных на кратных частотах по отношению к центральной частоте ПП, что не позволяет получать широкие полосы заграждения (ШПЗ). Традиционное применение дополнительных фильтров для расширения ПЗ сопровождается ростом потерь в ПП и ухудшением целого ряда тактикотехнических характеристик РТС, в состав которых входят подобные фильтрующие устройства. Существенно возрастают массо-габаритные показатели (особенно для многоканальных систем с ФАР), стоимость и т. д.

В связи с этим была поставлена научная задача, которая заключалась в разработке метода синтеза полосно-пропускающих фильтров СВЧ на неоднородных линиях, позволяющего осуществить выбор структуры и параметров ППФ, обеспечивающих максимальную протяженность полосы заграждения для выполнения требований ЭМС при заданных ограничениях на характеристики затухания в полосах пропускания и заграждения и установленных конструктивно-технологических ограничений по перепаду волновых сопротивлений и допускам на изготовление.

В процессе ее решения были разработаны методики и программы для ЭВМ:

1. Методика обоснования возможности увеличения протяженности полосы заграждения в ППФ на основе многоступенчатых линий [2, 3].

Методика построена на основе метода разрядки спектра резонансных частот (рис. 1) и позволяет:

при выборе структуры неоднородной линии учитывать новый комбинированный критерий, состоящий в получении максимальной разрядки спектра резонансных частот при минимальном числе ступенек;

определять основные параметры и эквивалентную схему резонатора в окрестности полюса входного сопротивления;

получать аналитические выражения и оценивать величину разрядки и протяженность полосы заграждения при изменении перепада волновых сопротивлений трехступенчатой разомкнутой линии по формуле 


$$
\mathrm{R}=\frac{\theta_{2 \mathrm{P}}}{\theta_{1}}=\frac{\pi-\operatorname{arctg} \xi_{1}}{\operatorname{arctg} \xi_{1}}=\frac{\pi}{\operatorname{arctg}\left(\sqrt{\left.(1+2 \operatorname{Pr}) / \mathrm{Pr}^{2}\right)}\right.}-1
$$

где $\theta_{2 p}$ - электрическая длина, соответствующая второй (ближайшей) полосе пропускания, $\theta_{2 \mathrm{p}}=\omega_{2 \mathrm{p}} \mathrm{t}_{3}=2 \pi f_{2 \mathrm{p}} \mathrm{t}_{3}, \mathrm{f}_{2 \mathrm{p}}-$ линейная частота второй (ближайшей) полосы пропускания, $\mathrm{t}_{3}-$ время задержки в линии; $\theta_{1}$ - электрическая длина ступеньки, соответствующая центральной частоте основной полосы пропускания фильтра, $\theta_{1}=\omega_{1 \mathrm{p}} \mathrm{t}_{3}=2 \pi \mathrm{f}_{1 \mathrm{p}} \mathrm{t}_{3}, \mathrm{f}_{1 \mathrm{p}}=\mathrm{f}_{0}$ - центральная частота основной полосы пропускания фильтра, $\theta_{2 \mathrm{p}} / \theta_{1}=\mathrm{R}$ - разрядка спектра резонансных частот или разрядка паразитных полос пропускания (ППП) - $\mathrm{R}=\mathrm{f}_{2} / \mathrm{f}_{0}, \mathrm{Pr}$ - перепад волновых сопротивлений, $\mathrm{Pr}=\mathrm{W}_{\max } / \mathrm{W}_{\min }$, где $\mathrm{W}_{\max }, \mathrm{W}_{\min }$ - максимальное и минимальное волновое сопротивление соответственно.

Результаты расчета по формуле (1) представлены в табл. 1.

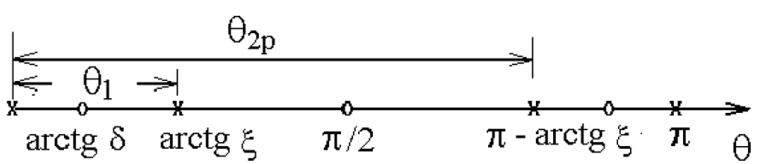

a)

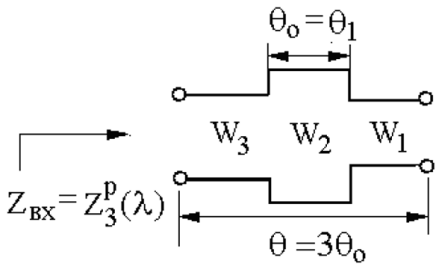

б)

Рис. 1. Распределение резонансных (крестики) и противорезонансных частот (кружочки) (а) и топологическая схема (б) трехступенчатой неоднородной линии

Fig. 1. Distribution of resonant (crosses) and anti-resonant frequencies (circles) (a) and topological diagram (б) of a three-step inhomogeneous line

Таблица 1. Зависимость разрядки спектра резонансных частот $\mathrm{R}=\theta_{2 \mathrm{p}} / \theta_{1}$ от перепада $\operatorname{Pr}$

Table 1. Dependence of the discharge of the spectrum of resonant frequencies $R=\theta_{2 p} / \theta_{1}$ on the difference $\operatorname{Pr}$

\begin{tabular}{|l|c|c|c|c|c|c|c|c|c|c|c|}
\hline \multicolumn{1}{|c|}{$\operatorname{Pr}$} & $\mathbf{2}$ & $\mathbf{3}$ & $\mathbf{4}$ & $\mathbf{5}$ & $\mathbf{6}$ & $\mathbf{7}$ & $\mathbf{8}$ & $\mathbf{9}$ & $\mathbf{1 0}$ & $\mathbf{1 1}$ & $\mathbf{1 2}$ \\
\hline$\theta_{2 \mathrm{p}} / \theta_{1}$ & 2.74 & 3.35 & 3.88 & 4.36 & 4.81 & 5.22 & 5.60 & 5.97 & 6.31 & 6.64 & 6.96 \\
\hline $\operatorname{Pr}$ & $\mathbf{1 3}$ & $\mathbf{1 4}$ & $\mathbf{1 5}$ & $\mathbf{1 6}$ & $\mathbf{1 7}$ & $\mathbf{1 8}$ & $\mathbf{1 9}$ & $\mathbf{2 0}$ & $\mathbf{2 1}$ & $\mathbf{2 2}$ & $\mathbf{2 3}$ \\
\hline$\theta_{2 \mathrm{p}} / \theta_{1}$ & 7.26 & 7.56 & 7.84 & 8.11 & 8.38 & 8.64 & 8.89 & 9.14 & 9.38 & 9.61 & 9.85 \\
\hline $\operatorname{Pr}$ & $\mathbf{2 4}$ & $\mathbf{2 5}$ & $\mathbf{2 6}$ & $\mathbf{2 7}$ & $\mathbf{2 8}$ & $\mathbf{2 9}$ & $\mathbf{3 0}$ & $\mathbf{3 1}$ & $\mathbf{3 2}$ & $\mathbf{3 3}$ & $\mathbf{3 4}$ \\
\hline$\theta_{2 \mathrm{p}} / \theta_{1}$ & 10.07 & 10.29 & 10.51 & 10.72 & 10.93 & 11.13 & 11.34 & 11.53 & 11.73 & 11.92 & 12.11 \\
\hline
\end{tabular}

\section{2. Методика синтеза ППФ на проходных трехступенчатых резонаторах с увеличен-} ной полосой заграждения [4-6].

Основу методики составляет подход Кона, который широко применяется при построении фильтрующих и согласующих устройств СВЧ на однородных линиях по параметрам низкочастотного прототипа. В отличие от известных методика позволяет: 
выбрать структуру фильтра и синтезировать ППФ, обеспечивающие требуемую разрядку паразитных полос пропускания для обеспечения ЭМС путем изменения перепада волновых сопротивлений трехступенчатой разомкнутой линии;

исследовать потенциальные характеристики ППФ и рассчитать их параметры (число резонаторов, их электрическую длину и волновые сопротивления).

Результаты моделирования представлены на рис. 2. Анализ зависимостей коэффициента затухания $\mathrm{L}$ от относительной частоты в полосе заграждения показывает, что увеличение перепада волновых сопротивлений Рr позволяет обеспечить необходимый разнос частот паразитных полос пропускания $\mathrm{R}$, причем значения $\mathrm{R}$ с точностью не хуже 5 \% совпадают с результатами расчета $\mathrm{R}$ по формуле (1). Моделирование показывает, что при перепадах $\operatorname{Pr}=2,5 ; 6,5 ; 12$ и 24 разнос частот составляет $\mathrm{R}=3,5,7$ и 10 соответственно.

3. Методика назначения допусков на параметры фильтров с иирокими полосами заграждения методом Монте - Карло [7, 8].

Суть методики состоит в многократном моделировании на ЭВМ передаточной функции фильтра (характеристики затухания) при различных комбинациях случайных отклонений параметров от своих номинальных значений. В отличие от известных методика позволяет:

получать при случайных отклонениях параметров ППФ СВЧ от своих номинальных значений зависимости среднеквадратических отклонений затухания в полосе пропускания (ПП) и полосе заграждения (ПЗ);

назначать допуски на изготовление с учетом процента выхода годных устройств на основе метода статистических испытаний Монте - Карло.

Для проведения натурного эксперимента использовался ППФ, представляющий собой трехрезонаторную структуру с трехступенчатыми резонаторами и параметрами:

центральная частота полосы пропускания $\mathrm{f}_{0}=800$ МГц; относительная ширина полосы пропускания $\mathrm{w}=0,05$ (абсолютная $40 \mathrm{MГц);}$

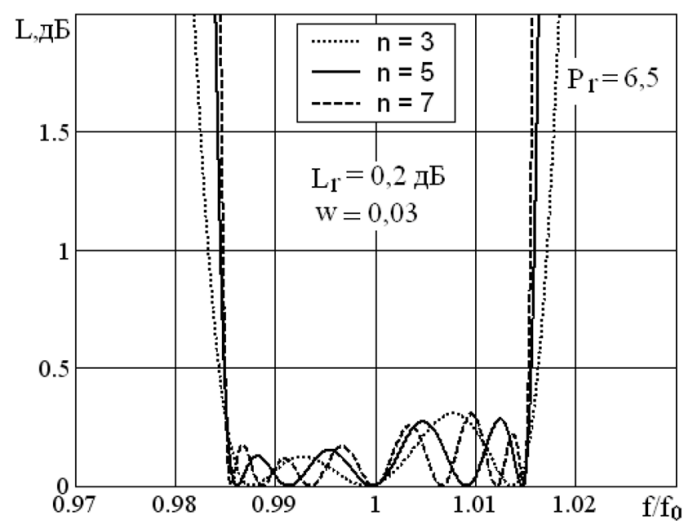

a)

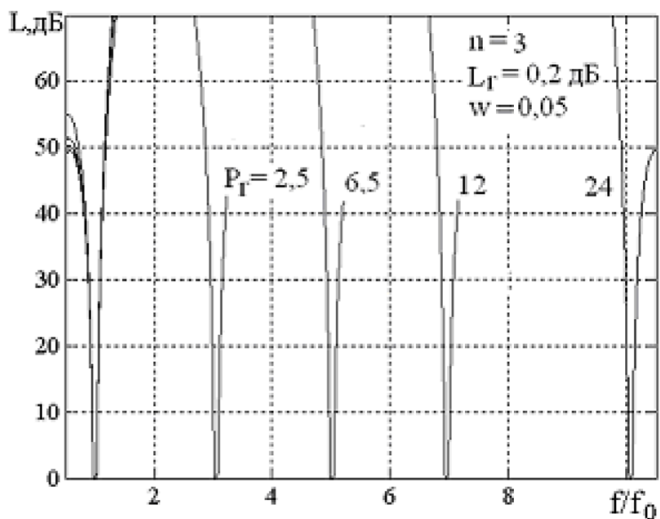

б)

Рис. 2. Зависимость коэффициента затухания L от относительной частоты в полосе пропускания (а) многорезонаторных и полосе заграждения для трехрезонаторного ППФ (б) при различном перепаде волновых сопротивлений $\mathrm{Pr}$

Fig. 2. Dependence of the attenuation coefficient L on the relative frequency in the passband (a) multi-resonator and stopband for a three-resonator PPF (б) with different drop in characteristic impedances $\operatorname{Pr}$ 
уровень пульсаций чебышевской характеристики затухания $\mathrm{Lr}=0,5$ дБ;

волновые сопротивления подводящих линий $\mathrm{W}=20$ Ом;

перепад волновых сопротивлений $\mathrm{Pr}=3$ (разнос ППП составляет $\mathrm{R}=3,35$ ).

Опытный образец ППФ (рис. 3а) был изготовлен по промышленной технологии фотохимическим методом на основе фольгированного материала Флан-2,8 (толщина пластины 2 мм) с параметрами:

относительная диэлектрическая проницаемость подложки $\varepsilon_{\mathrm{r}}=2,8$;

толщина симметричной полосковой линии $\mathrm{b}=4$ мм.

Натурный эксперимент проводили по стандартной методике на приборе фирмы Agilent E5071C - анализатор цепей (рис. 3б).

Сравнение измеренных (рис. Зг) и рассчитанных характеристик фильтра (рис. Зв) показало следующее:

в полосе заграждения (ПЗ) на кратных частотах по сравнению с существующими ППФ с емкостными зазорами обеспечивается повышенное затухание - на частоте 1,6 ГГц (первая паразитная полоса пропускания) более 100 дБ;

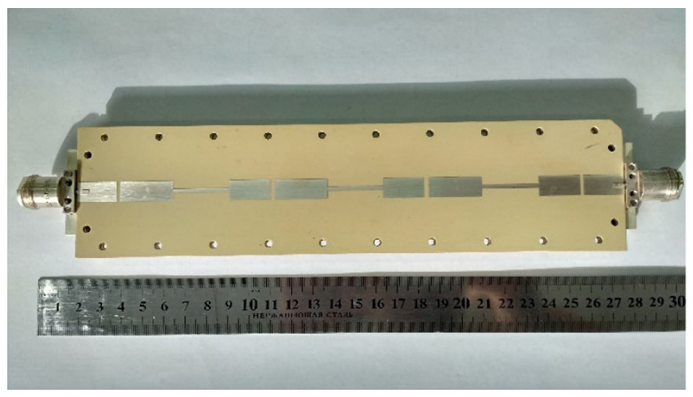

a)

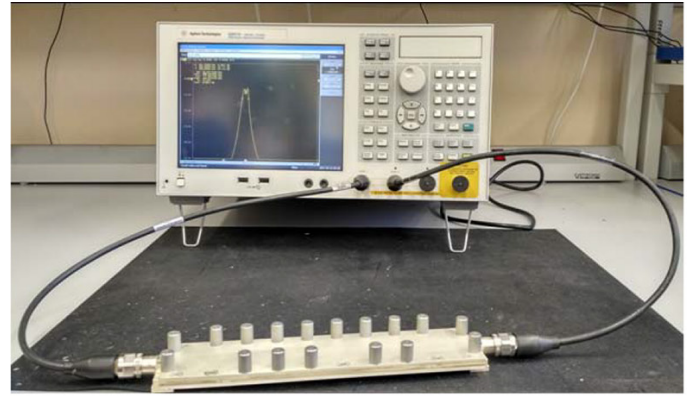

б)

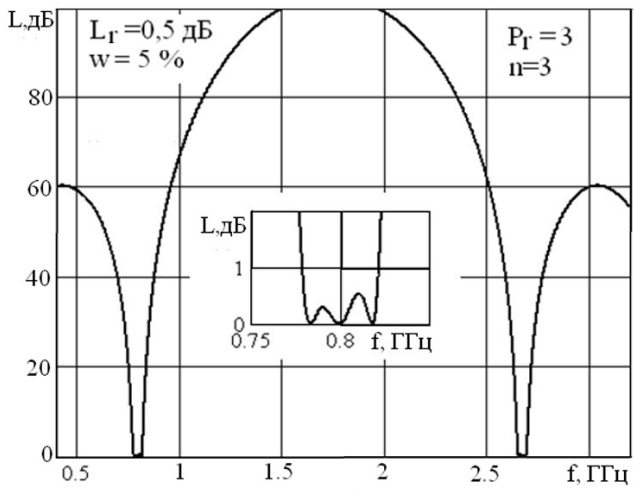

B)

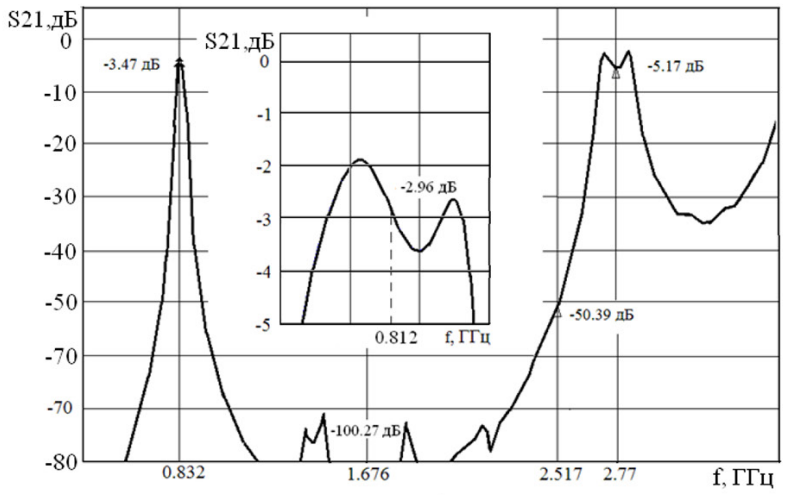

Г)

Рис. 3. Внешний вид трехрезонаторного фильтра на проходных трехступенчатых линиях со снятой верхней пластиной (а), вид экспериментальной установки (б), результаты математического моделирования характеристик затухания фильтра в полосе пропускания и заграждения (в) и измеренной характеристики $(\mathrm{AЧХ)} \mathrm{(г)}$

Fig. 3. Appearance of a three-resonator filter on three-stage pass-through lines with the upper plate removed (a), the view of the experimental setup (б), the results of mathematical modeling of the filter attenuation characteristics in the passband and barriers (в) and the measured characteristic (AFC) (Г) 
на частоте 2,4 ГГц (вторая паразитная полоса пропускания) - 70 дБ. Сравнение с измерениями показывает, что на данных частотах затухание составляет от 60 до 70 дБ;

первая паразитная полоса пропускания составляет 2,77 ГГц (при основной частоте полосы пропускания 814 МГц), что обеспечивает разнос резонансных частот $\mathrm{R}=1: 3,4$, что с точностью 1,47 \% соответствует результату математического моделирования и расчетам по формуле (1) 1:3,35 (при основной частоте полосы пропускания 800 МГц).

Результаты измерения показали, что центральная частота полосы пропускания - 814 МГц больше задаваемой при расчете на 14 МГц (1,75\%), а полоса пропускания - 38,65 МГц, что меньше на 1,35 МГц (3,4 \%). Наблюдается ее смещение в область высоких частот. Отличие расчетных и экспериментальных характеристик обусловлено отсутствием учета потерь и высших типов волн при моделировании ППФ, а также наличием погрешностей при изготовлении конструкции ППФ.

Таким образом, экспериментальное исследование опытного образца ППФ с ШПЗ позволило подтвердить правильность подхода к разработке ППФ на неоднородных линиях, обеспечивающего заданную полосу заграждения путем изменения перепада волновых сопротивлений трехступенчатых линий.

\section{Список литературы / References}

[1] Бердышев Р. В., Журавлев А. В., Цыганов А. А., Парфенов К. В., Панкрушин В. Н., Короченцев О.Н. Оценка качества применения ППФ на неоднородных линиях в приемо-передающем тракте РЛС 67Н6Е. Сборник научно-методических трудов международной ВНК, посвященной 60-летию академии (2-3 марта 2017 г.). Тверь: ВА ВКО, 2017, 46-52. [Berdyshev R.V., Zhuravlev A. V., Tsyganov A. A., Parfenov K. V., Pankrushin V.N., Korochentsev O. N. Assessment of the quality of PPF application on non-uniform lines in the transmit-receive path of the 67N6E radar. Collection of scientific and methodological works of the international VNK, dedicated to the 60th anniversary of the Academy (March 2-3, 2017). Tver, VA VKO, 2017, 46-52 (in Russian)]

[2] Бердышев В.П., Бердышев Р. В., Пантилеев В. В., Короченцев О.Н. Методика обоснования структуры радиопрозрачных укрытий и фильтров СВЧ с увеличенной полосой заграждения. Научно-методический сборник материалов I международной ВНК (1-2 апреля 2015 г.). Секция 4. Т. 6. Тверь: ВА ВКО, 2015, 187-191. [Berdyshev V.P., Berdyshev R. V., Pantileev V.V., Korochentsev O.N. Methodology for substantiating the structure of radio-transparent shelters and microwave filters with an increased barrage. Scientific and methodological collection of materials of the I international VNK (April 1-2, 2015). Section 4. Vol. 6. Tver, VA VKO, 2015, 187-191 (in Russian)]

[3] Бердышев В.П., Журавлев А.В., Бердышев Р.В., Короченцев О.Н., Панкрушин В.Н., Пантилеев В.В. Программа синтеза и оптимизации параметров неоднородных многоступенчатых линий по заданному распределению резонансных и противорезонансных частот для построения устройств СВЧ с улучшенными частотными характеристиками. Свидетельство о государственной регистрации программ для ЭВМ № 2015662275. Заявка № 2015619002, дата поступ. 22.09.15, дата регистрац. 19.11.2015. М.: ФГУ ФИПС, 2015. [Berdyshev V.P., Zhuravlev A. V., Berdyshev R. V., Korochentsev O.N., Pankrushin V.N., Pantileev V.V. Program for the synthesis and optimization of the parameters of non-uniform multistage lines for a given distribution of resonant and anti-resonant frequencies for the construction of microwave devices with improved frequency 
characteristics. Certificate of state registration of computer programs № 2015662275. Application № 2015619002, date of receipt. 22.09.15, registration date. 19.11.2015. M., FGU FIPS, 2015 (in Russian)]

[4] Бердышев В.П., Бердышев Р. В., Журавлев А. В., Пантилеев В. В., Панкрушин В. Н., Короченцев О.Н. Синтез проходных ступенчатых линий для построения согласующих устройств и полосно-пропускающих фильтров СВЧ в интересах обеспечения требований ЭМС. Сборник докладов XVI ВНК. Ярославль: ЯВВИУ ПВО, 2015. С. 3-11. [Berdyshev V.P., Berdyshev R.V., Zhuravlev A.V., Pantileev V.V., Pankrushin V.N., Korochentsev O.N. Synthesis of pass-through stepped lines for the construction of matching devices and microwave bandpass filters in the interests of meeting the EMC requirements. Collection of reports of the XVI VNK. Yaroslavl, JAVVIU Air Defense, 2015. P. 3-11 (in Russian)]

[5] Бердышев В.П., Журавлев А.В., Бердышев Р. В., Короченцев О.Н., Панкрушин В.Н. Программа расчета частотных характеристик полосно-пропускающих фильтров СВЧ на проходных трехступенчатых резонаторах с широкой полосой заграждения. Свидетельство о государственной регистрации программ для ЭВМ № 2015662305. Заявка № 2015619180, дата поступ. 22.09.15, дата регистрац. 19.11.2015. М.: ФГУ ФИПС, 2015. [Berdyshev V.P., Zhuravlev A. V., Berdyshev R. V., Korochentsev O.N., Pankrushin V.N. Program for calculating the frequency characteristics of microwave bandpass filters on three-stage pass-through resonators with a wide stop band. Certificate of state registration of computer programs № 2015662305. Application № 2015619180, date of receipt. 22.09.15, registration date 19.11.2015. M., FGU FIPS, 2015 (in Russian)]

[6]Бердышев В. П.,Журавлев А. В., Бердышев Р. В., Короченцев О. Н.,Панкрушин В. Н. Программа расчета частотных характеристик многорезонаторных полосно-пропускающих фильтров и согласующих устройств СВЧ с емкостными зазорами на проходных трехступенчатых резонаторах с заданной полосой заграждения. Свидетельство о государственной регистрации программ для ЭВМ № 2015661742. Заявка № 2015619016, дата поступ. 22.09.15, дата регистрац. 06.11.2015. М.: ФГУ ФИПС, 2015. [Berdyshev V.P., Zhuravlev A.V., Berdyshev R.V., Korochentsev O.N., Pankrushin V.N. Program for calculating the frequency characteristics of multiresonator band-pass filters and microwave matching devices with capacitive gaps on three-stage passthrough resonators with a given stop band. Certificate of state registration of computer programs № 2015661742. Application № 2015619016, date of receipt. 22.09. 15, registration date 06.11. 2015. M.: FGU FIPS, 2015 (in Russian)]

[7] Бердышев Р.В., Панкрушин В.Н., Короченцев О.Н. Методика назначения допусков на параметры фильтров с широкими полосами заграждения методом Монте - Карло. Сборник научных статей по материалам IV Всероссийской научно-практической конференции «Академические Жуковские чтения» (23-24 ноября 2016 г.). Воронеж: ВУНЦ ВВС «ВВА», 2017. С. 2832. [Berdyshev R. V., Pankrushin V.N., Korochentsev O.N. Technique for assigning tolerances to the parameters of filters with wide stopbands using the Monte Carlo method. Collection of scientific articles based on the materials of the IV All-Russian Scientific and Practical Conference "Academic Zhukov Readings» (November 23-24, 2016). Voronezh, VUNC VVS «VVA», 2017. P. 28-32 (in Russian)]

[8] Бердышев В.П., Журавлев А.В., Бердышев Р.В., Короченцев О.Н., Панкрушин В.Н., Полтавский Н.А., Парфенов К.В. Программа расчета допусков на параметры полоснопропускающих фильтров СВЧ на проходных трехступенчатых резонаторах с широкой полосой заграждения методом Монте-Карло. Свидетельство о государственной регистрации программ 
для ЭВМ № 2017610134. Заявка № 2016662074, дата поступ. 08.11.16, дата регистр. 09.01.2017. М.: ФГУ ФИПС, 2017. [Berdyshev V.P., Zhuravlev A.V., Berdyshev R.V., Korochentsev O.N., Pankrushin V.N., Poltavsky N. A., Parfenov K.V. A program for calculating the tolerances for the parameters of microwave bandpass filters on three-stage pass-through resonators with a wide stop band by the Monte Carlo method. Certificate of state registration of computer programs № 2017610134. Application № 2016662074, date of receipt. 08.11.16, register date 09.01.2017. M., FGU FIPS, 2017 (in Russian)] 